\title{
Perturbing the metabolic dynamics of myo-inositol in developing Brassica napus seeds through in vivo methylation impacts its utilization as phytate precursor and affects downstream metabolic pathways
}

Jinzhuo Dong ${ }^{1 \dagger}$, Wei Yan² ${ }^{2 \dagger}$ Cheryl Bock², Kateryna Nokhrina ${ }^{2}$, Wilf Keller ${ }^{3}$ and Fawzy Georges ${ }^{2 *}$

\begin{abstract}
Background: myo-Inositol (Ins) metabolism during early stages of seed development plays an important role in determining the distributional relationships of some seed storage components such as the antinutritional factors, sucrose galactosides (also known as raffinose oligosaccharides) and phytic acid (PhA) (myo-inositol 1,2,3,4,5,6hexakisphosphate). The former is a group of oligosaccharides, which plays a role in desiccation at seed maturation. They are not easily digested by monogastric animals, hence their flatulence-causing properties. Phytic acid is highly negatively charged, which chelates positive ions of essential minerals and decreases their bioavailability. It is also a major cause of phosphate-related water pollution. Our aim was to investigate the influence of competitive diversion of Ins as common substrate on the biosynthesis of phytate and sucrose galactosides.

Results: We have studied the initial metabolic patterns of Ins in developing seeds of Brassica napus and determined that early stages of seed development are marked by rapid deployment of Ins into a variety of pathways, dominated by interconversion of polar (Ins phosphates) and non-polar (phospholipids) species. In a time course experiment at early stages of seed development, we show Ins to be a highly significant constituent of the endosperm and seed coat, but with no phytate biosynthesis occurring in either tissue. Phytate accumulation appears to be confined mainly within the embryo throughout seed development and maturation. In our approach, the gene for myo-inositol methyltransferase (IMT), isolated from Mesembryanthemum crystallinum (ice plant), was transferred to B. napus under the control of the seed-specific promoters, napin and phaseolin. Introduction of this new metabolic step during seed development prompted Ins conversion to the corresponding monomethyl ether, ononitol, and affected phytate accumulation. We were able to produce homozygous transgenic lines with 19\% - 35\% average phytate reduction. Additionally, changes in the raffinose content and related sugars occurred along with enhanced sucrose levels. Germination rates, viability and other seed parameters were unaffected by the IMT transgene over-expression.

Conclusions: Competitive methylation of Ins during seed development reduces seed antinutritional components and enhances its nutritional characteristics while maintaining adequate phosphate reserves. Such approach should potentially raise the canola market value and likely, that of other crops.
\end{abstract}

Keywords: Brassica napus, Seed coat, Protein translation, Ononitol, Seed development, myo-Inositol methyltransferase, Phytic acid, Sucrose, Raffinose oligosaccharides, Galactinol

\footnotetext{
*Correspondence: fawzy.georges@nrc-cnrc.gc.ca

${ }^{\dagger}$ Equal contributors

${ }^{2}$ National Research Council Canada, Plant Biotechnology Institute, 110

Gymnasium Place, Saskatoon, SK S7N 0W9, Canada

Full list of author information is available at the end of the article
} 


\section{Background}

myo-Inositol is an essential component in the biosynthesis of an array of derivatives ranging from simple inositol phosphates to complex membrane-associated products with important cellular functions. It can be isomerized and (or) methylated to form a variety of species-specific epimers and methyl ethers. A number of these, including Ins, have been noted to accumulate mostly in osmotically challenged plants and have since been recognized as osmoprotectant metabolites [1].

Ins is also central to the biosynthesis of a number of antinutritional components such as sucrose galactosides (e.g. RFO) and inositol polyphosphates such as PhA (also known as Ins $_{6}$ ). The synthesis of PhA predominates in developing seeds, and constitutes the major storage form of seed phosphorus. Both Ins and its bound phosphates are released by hydrolysis upon germination.

The antinutritional properties of $\mathrm{PhA}$ reside in its strong binding affinity for positively charged species such as essential minerals (e.g. iron and zinc) and proteins, significantly lowering their bioavailability to humans and animals. As a consequence, the presence of high levels of $\mathrm{PhA}$ in canola seed hinders the full exploitation of the pure meal and underrates its potential as a major crop worldwide.

In contrast, PhA has been accredited as an effective antioxidant with antitumor properties and risk reduction of certain types of cancer [2]. Besides decreasing uncontrolled cellular proliferation, $\mathrm{PhA}$ is also thought to cause differentiation of malignant cells resulting in reversion to the normal phenotype [3]. Further, PhA has been shown to play a critical role in many cellular events such as signaling [4], apoptosis [5,6], neuroprotection [7], as well as functioning as enzyme cofactor [8]. The antioxidant properties of $\mathrm{PhA}$ have also been shown to inhibit free radical formation and lower lipid peroxidation, making it a very efficient natural food and feed preservative [9]. Moreover, PhA has been shown recently to protect developing seeds against oxidative stress [10]. Because of the seemingly paradoxical and unique roles of $\mathrm{PhA}$, and in view of the agronomic value placed on low phytate-containing seeds and (or) meals, total elimination of PhA was not our intended goal.

Phytic acid biosynthesis constitutes a uniquely complex process, consisting of a primary substrate, Ins, and a number of interjecting secondary Ins polyphosphate substrates from other sources and pathways [11,12]. In the primary pathway, the de novo synthesis of Ins involves the oxidative cyclization of glucose 6-phosphate (G-6-P) to L-myo-inositol-1-phosphate (L-Ins-1-P) by the action of a single enzyme, L-myo-inositol-1-phosphate synthase (MIPS). It has been shown that by affecting the production of this enzyme directly or indirectly through mutagenesis in maize $[13,14]$ and soybean [15] or by genetic engineering methods in rice $[16,17]$, soybean [18] and canola [19] $\mathrm{PhA}$ accumulation can be reduced by $20-94.5 \%$ with a concomitant increase in inorganic phosphate $\left(\mathrm{P}_{\mathrm{i}}\right)$. Of the various transgenic approaches reported, MIPS-RNAi [18] and MIPScosuppression [19] transgenics yielded the lowest levels of $\mathrm{PhA}$ in the corresponding mature seeds (94.5 and $44 \%$, respectively). However, the RNAi approach reportedly has resulted in hindered seed development [18]. Thus, in view of the fact that Ins is a key substrate in the biosynthesis of many essential cell components, we decided to assess the consequences of its metabolic diversion versus its complete elimination through MIPS down-regulation, and compare the effects associated with each approach. We reasoned that while metabolic diversion of Ins may not interfere with its biosynthesis, it could preferably limit its participation in PhA production.

The first MIPS product, L-Ins-1-P, appears to be in quasi equilibrium with free Ins due to the activity of a two-enzyme system, in which L-myo-inositol-1-phosphate monophosphatase converts L-Ins-1-P to free Ins, while myo-inositol-1-kinase regenerates it (Scheme 1). Depending on the rigidity of the requirements for free Ins $v s$. L-Ins-1-P in the developing seed, the relative abundance of each substrate is ultimately determined by the differences in the kinetics of the two opposing reactions. We hypothesized that continual exclusion of free Ins from this quasi equilibrium by metabolic diversion through methylation, could drive the quasi equilibrium in the direction of free Ins. This would subsequently limit the participation of both substrates (Ins and L-Ins1-P) in PhA biosynthesis. Towards this end, we studied the effect of over-expressing the gene encoding IMT (EC 2.1.1.129) from M. crystallinum [20] on seed PhA accumulation in transgenic $B$. napus under two different seed-specific promoters, napin and phaseolin [21]. We also examined the effect of enhancing the translational context of IMT on its gene product accumulation and PhA reduction during seed development.

Although the M. crystallinum IMT (McIMT) has been used to study the osmoprotective properties of methylated cyclitols in transgenic plants [22-25], the current investigation is the first example of seed-specific McIMT-mediated metabolic diversion to reduce phytic acid biosynthesis in seed crops through in vivo methylation of Ins.

\section{Results}

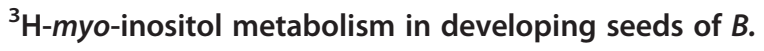
napus

In vivo labeling of developing $B$. napus seeds with ${ }^{3} \mathrm{H}-m y o$-inositol, and subsequent fractionation of different cell components (acid-soluble, hexane-soluble, trifluoroacetic acid [TFA]-soluble) and cell debris revealed the 


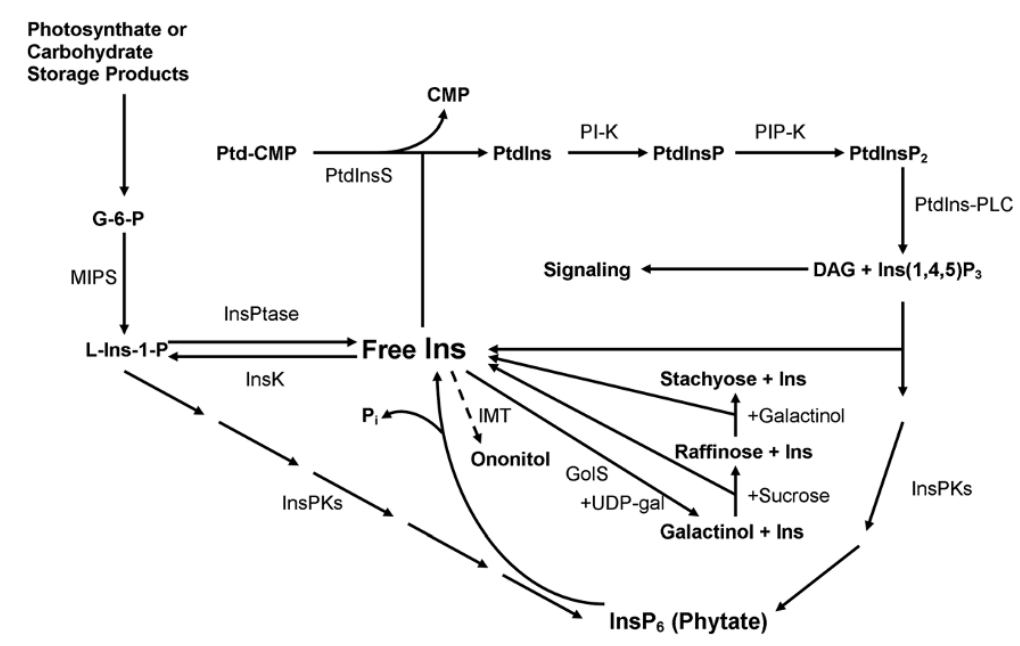

Scheme 1 Metabolic interconversions of phosphorylated myo-inositol and related derivatives in developing B. napus seeds. Dashed arrow indicates the newly introduced methylation step. Solid lines represent established metabolic pathways. PI-K, phosphatidylinositol kinase; GolS, galactinol synthase; MIPS, myo-inositol phosphate synthase; InsPKs, myo-inositol kinases; PtdlnsS, Ptdlns synthase; Ptdlns-PLC, Ptdlns-specific phospholipase C; InsPtase, myo-inositol phosphate phosphatase; InsK, myo-inositol kinase; G-6-P, glucose-6-phosphate; DAG, diacylglycerol; UDP-gal, uridine diphosphate galactose; Ptd-CMP, phosphatidylcytosine monophosphate; Ptdlns, phosphatidylinositol; PtdlnsP, phosphatidylinositol monophosphate; $\mathrm{P}_{\mathrm{i}}$, inorganic phosphate.

relative incorporation of ${ }^{3} \mathrm{H}$-myo-inositol in each fraction (Figure 1). The acid-soluble fraction contains free Ins, Ins monophosphates and Ins polyphosphates. The hexanesoluble fraction consists mainly of Ins-containing phospholipids. The TFA-soluble fraction and cell debris mainly include tightly bound membrane components such as glycosyl-phosphatidyl inositol (GPI) protein anchors [26]. Between 15 and 20 DAP, most of the label was recovered in the acid soluble fraction, which contains PhA. After 20 DAP, a decrease in the relative content of the acid-soluble ${ }^{3} \mathrm{H}$-myo-inositol-labeled fraction occurred until at least 30

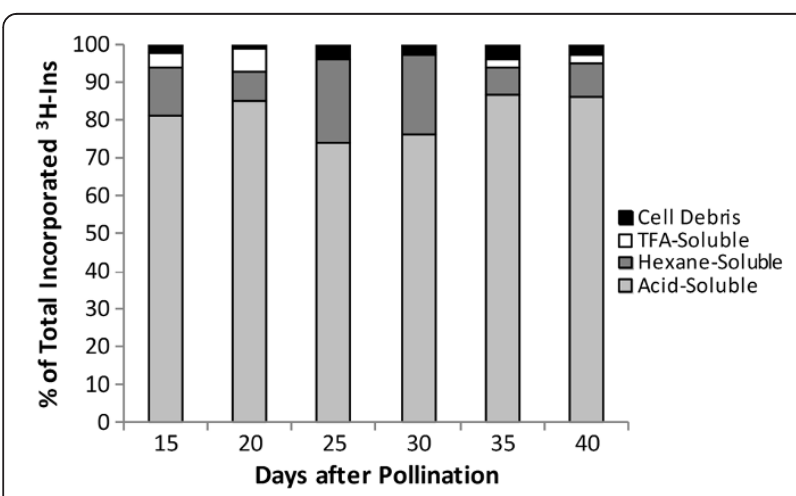

Figure 1 Differential extraction of ${ }^{3} \mathrm{H}$-myo-inositol incorporated in developing seeds. Developing seeds of $B$. napus were labeled with ${ }^{3} \mathrm{H}$-myo-inositol $\left({ }^{3} \mathrm{H}-\mathrm{Ins}\right)$ and different fractions were extracted as described in Methods. Data represent the percentage of total incorporated ${ }^{3} \mathrm{H}$-Ins in cell debris (black); TFA, (trifluoroacetic acid) (white); hexane (dark grey) and hydrochloric acid (light grey).
DAP. A simultaneous increase in the relative amount of label incorporation appeared in the corresponding hexanesoluble fraction. After $30 \mathrm{DAP}$, the relative rates of incorporation in the acid-soluble fraction increased again and remained high until at least $40 \mathrm{DAP}$.

\section{Phytic acid accumulation in developing seeds of Brassica napus}

HPLC analysis profiles indicate that PhA started to accumulate in detectable amounts during very early stages of seed development (12 DAP). Its accumulation became more pronounced at $20 \mathrm{DAP}$ and continued progressively, reaching maximum levels at about 35 DAP (Figure 2). This time window (12-35 DAP) is, therefore, important in temporal targeting of molecular strategies for phytic acid reduction in canola seeds. In spite of the fact that Ins was shown to be present in both endosperm and seed coat at all stages examined (Figure 3B), no phytate accumulation was found in either tissue (Figure 2).

Variations in myo-inositol levels during seed development At early stages of seed development, a steady decline in Ins levels occurred from 5 DAP up to 25 DAP (Figure 3A). These levels continued to decline further at 30 DAP, reaching their lowest point at 45 DAP through maturity. The sharp decline in the levels of Ins during the period 5-20 DAP coincided with the initial gradual accumulation of $\mathrm{PhA}$ (Figure 2) as well as the increase in the levels of the non-polar derivatives (Figure 1 at 15-30 DAP). At 30 DAP the rapid decline in Ins levels resumed with a 


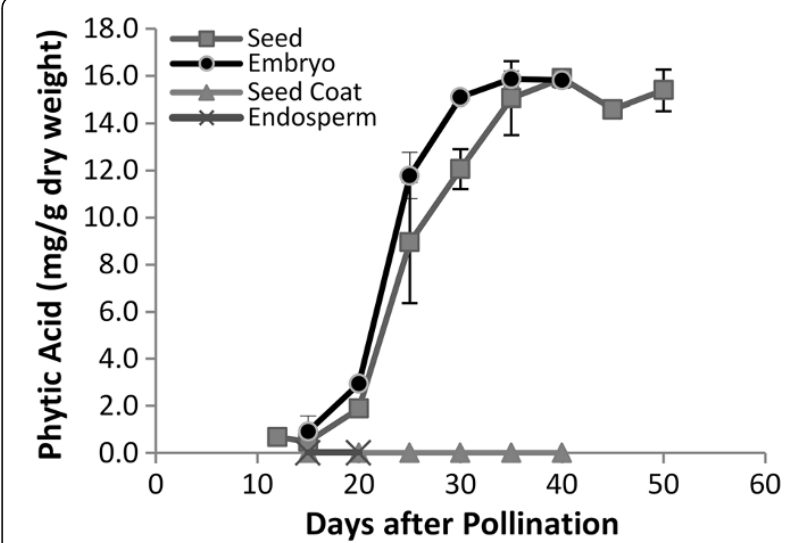

Figure 2 Phytic acid accumulation during $B$. napus seed development in whole and dissected seed. The level of phytic acid (PhA) was measured by HPLC in Westar whole seed, seed coat, endosperm and embryo at different developmental stages from 12 to 50 days after pollination (DAP). Each data point represents mean value of three biological replicates \pm SE (standard error). concomitant rise in PhA levels (Figure 2) at the expense of the non-polar components (Figure 1).

Meanwhile, as the embryo continued to expand through the different stages (Figure 3C), the level of Ins declined from its highest point in seed coat and endosperm and gradually increased in embryo tissues starting at 15-20 DAP (Figure 3B). At this point the rate of PhA biosynthesis in the embryo began to increase (Figure 2). No PhA synthesis was observed in either seed coat or endosperm.

Generation of transgenic lines of $B$. napus carrying the myo-inositol methyltransferase gene

Transgenic lines were generated from constructs pNIMT (IMT under napin promoter) and pPhIMT (IMT under phaseolin promoter). In both napin and phaseolin groups of transgenics, $80 \%$ of the lines showed reduced levels of $\mathrm{PhA}$. Three transgenic lines with the highest PhA reduction were chosen from each promoter group for subsequent experiments. These were selfed to homozygosity and were shown to be consistently stable in terms of the $I M T$ gene integrity and phytate reduction. One line from each group was chosen for further studies, namely $\mathrm{N}-11$
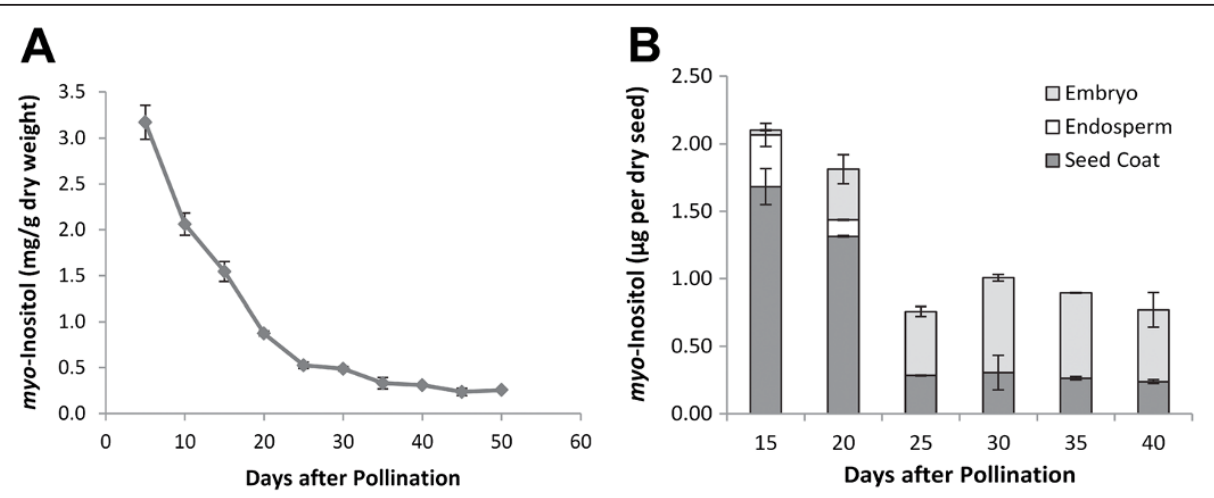

C
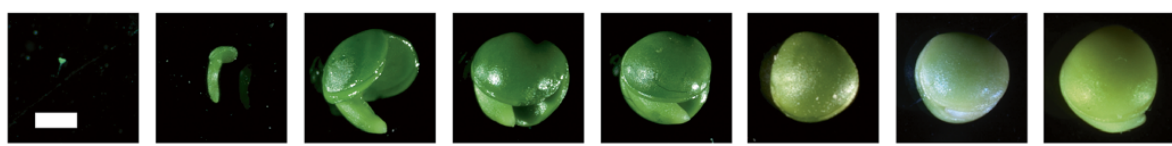

10 DAP

15 DAP 20 DAP 25
(Torpedo) (Bent Cotyledon)
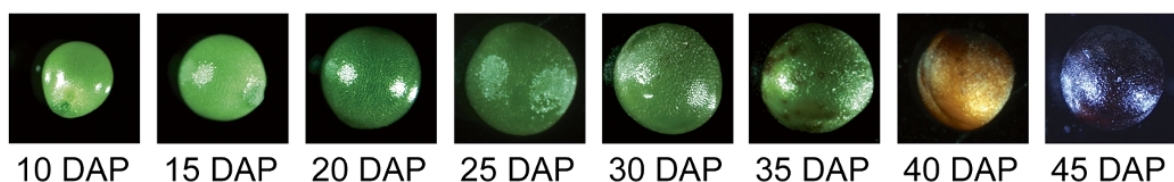

Figure 3 Analysis of myo-Inositol in whole and dissected seed at different developmental stages. (A) Declining levels of myo-Inositol (Ins) during B. napus seed development expressed as Ins content of whole seed. Ins was analyzed in Westar seed at different developmental stages from 5 to 50 days after pollination (DAP). Each data point represents mean value of three biological replicates \pm SE (standard error). (B) myo-Inositol distribution in different tissues of developing seed. Ins was measured in seed coat, endosperm and embryo at different developing stages. Data represent the amount of Ins distributed in seed coat (dark grey column), endosperm (white column) and embryo (light grey column) of one single seed. Each data point represents mean value of three biological replicates \pm SE (standard error). (C) Relative sizes of the developing embryo (top row) relative to seed coat (bottom row) at various stages of seed development. The white bar represents the length of $1 \mathrm{~mm}$. 
(napin) and Ph1-18 (phaseolin). Lines Ph2-15 and Ph3-19 originated from two more transformation events and were chosen in the same way. These lines differed from Ph1-18 in that they harbored changes in the translational context of the IMT gene (Table 1). In all transgenic lines germination rate was $100 \%$ for fresh seed, which did not differ from Westar controls. Additionally, over several generations the IMT transgenics did not exhibit changes in seed yield.

\section{IMT expression occurs progressively in developing seeds} of transgenic $B$. napus

In both lines of transformants (using napin and phaseolin promoters) production of IMT was verified by Western blot analysis, which also revealed absence of any native equivalents of IMT in developing seeds of nontransformed B. napus (Figure 4A and 4B). Although the $I M T$ transcript started to appear at 20 DAP in developing transgenic seeds as exemplified by the phaseolin lines (Figure 4A), Western-blot analysis showed the corresponding protein to be produced in detectable amounts only after 25 DAP. The accumulation pattern of both the $I M T$ transcript and its corresponding protein were similar in that they increased progressively to 40 DAP. Likewise, the napin-IMT line exhibited a similar manner of protein expression at 25 DAP (Figure 4B).

Transgenic seeds produce enzymatically active IMT and D-ononitol

HPLC analysis of mature transgenic seeds revealed the presence and accumulation of a new compound, which co-eluted with authentic D-ononitol standard (Figure 5A and $5 \mathrm{~B}$ ). The newly introduced IMT activity in transgenic lines was further confirmed by the ability of total soluble protein extracts from 40-DAP transgenic seeds to convert Ins to D-ononitol, in an in vitro IMT enzymatic assay (Figure 5C). Similar extracts from wild-type seeds as well as from transgenic leaves failed to produce this product. In addition, an unidentified compound eluted immediately prior to the Ins peak in the transgenic mature seed samples (not shown), which was absent in the wild-type samples. This compound did not co-elute with an authentic sample of pinitol.

Table 1 Modified translational contexts for IMT gene driven by the phaseolin promoter

\begin{tabular}{lll}
\hline Name of construct & Line & Sequence \\
\hline pPhIMT1 (parent-transgenic IMT) & Ph1-18 & $\mathrm{A}^{-3} \mathrm{~A}^{-2} \mathrm{~A}^{-1}$ ATG A \\
pPhIMT2 & Ph2-15 & $\mathrm{G}^{-3} \mathrm{C}^{-2} \mathrm{C}^{-1}$ ATG A \\
pPhIMT3 & Ph3-19 & $\mathrm{A}^{-3} \mathrm{C}^{-2} \mathrm{C}^{-1}$ ATG A \\
\hline
\end{tabular}

Sequence column shows DNA triplets 5 ' upstream from the translation initiation codon: line Ph1-18 (parent IMT) with dA nucleotides in positions -1 to -3 ; line Ph2-15 with dC nucleotides in positions -1 and -2 and dG nucleotide in position -3; line Ph3-19 with $\mathrm{dC}$ nucleotides in positions -1 and -2 and $\mathrm{dA}$ nucleotide in position -3 .

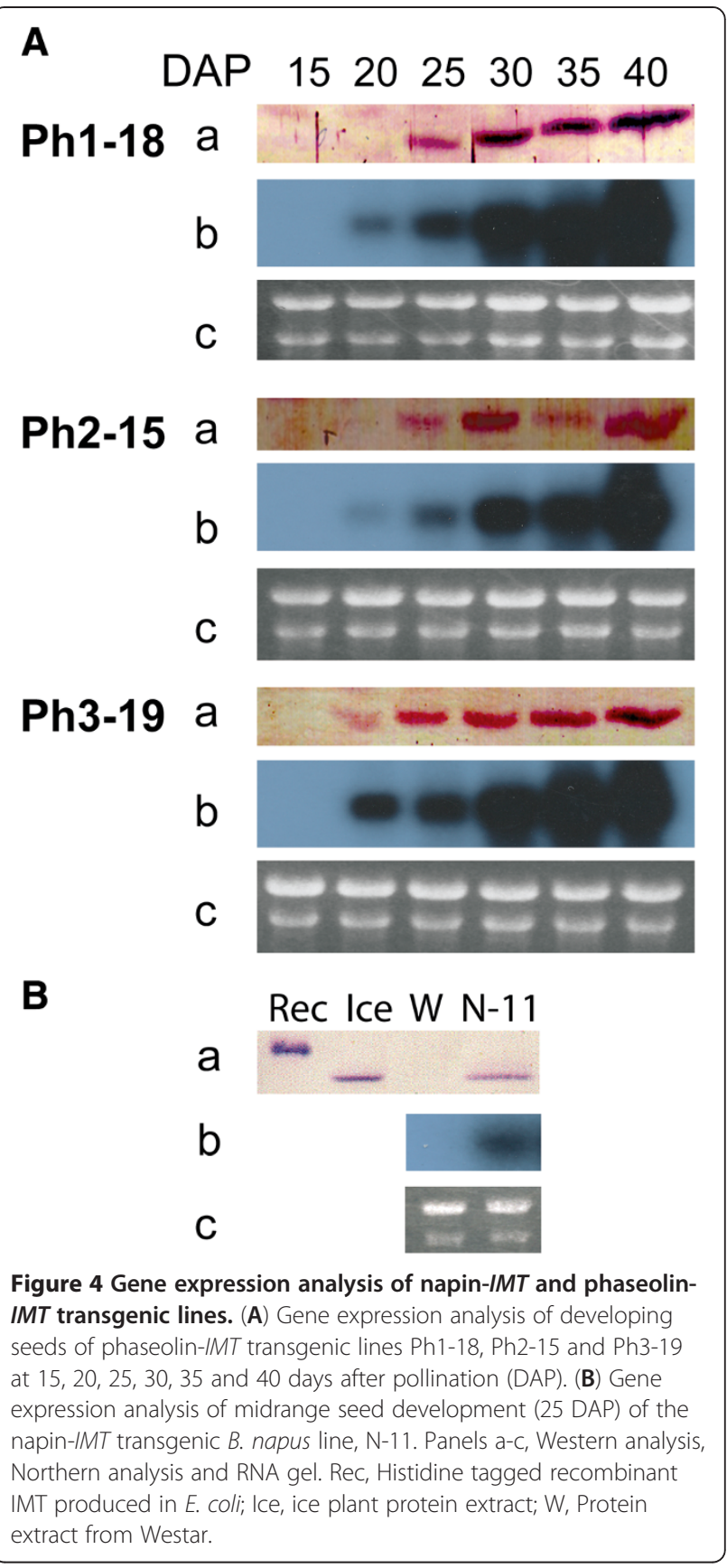

Phytic acid content is reduced and inorganic phosphate content is enhanced in the seeds of transgenic plants HPLC analysis for phytate content in mature napinIMT transgenic seeds showed that a $35 \%$ reduction in PhA level was achieved. HPLC analysis also showed a reduction of $19-29 \%$ in $\mathrm{PhA}$ in mature phaseolin-IMT seeds despite the translational context modification (Figure 6). Additionally, $\mathrm{P}_{\mathrm{i}}$ levels increased from 10 to $31 \%$ which is consistent with earlier observations (Figure 7). 


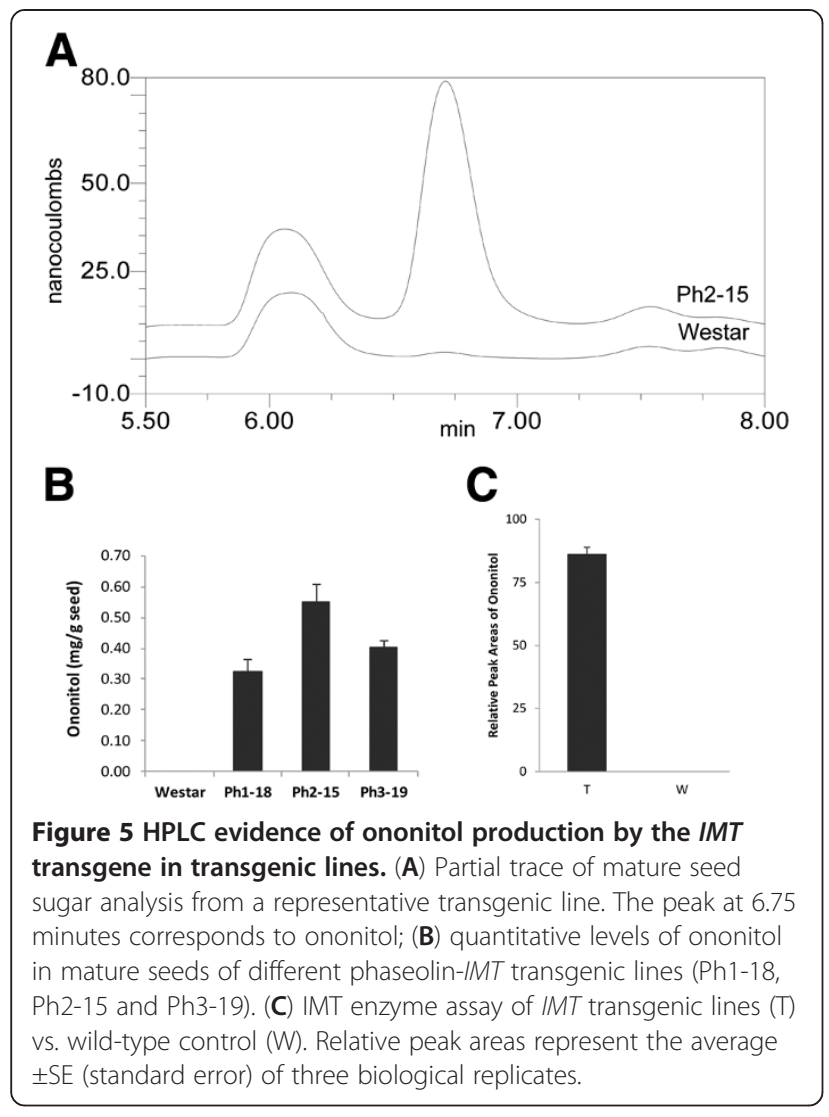

\section{Carbohydrate analysis}

Changes in the carbohydrate species associated with Ins metabolism (e.g. galactinol, RFO and sucrose) were observed in mature transgenic seeds. In the three lines examined, the changes were consistent with the

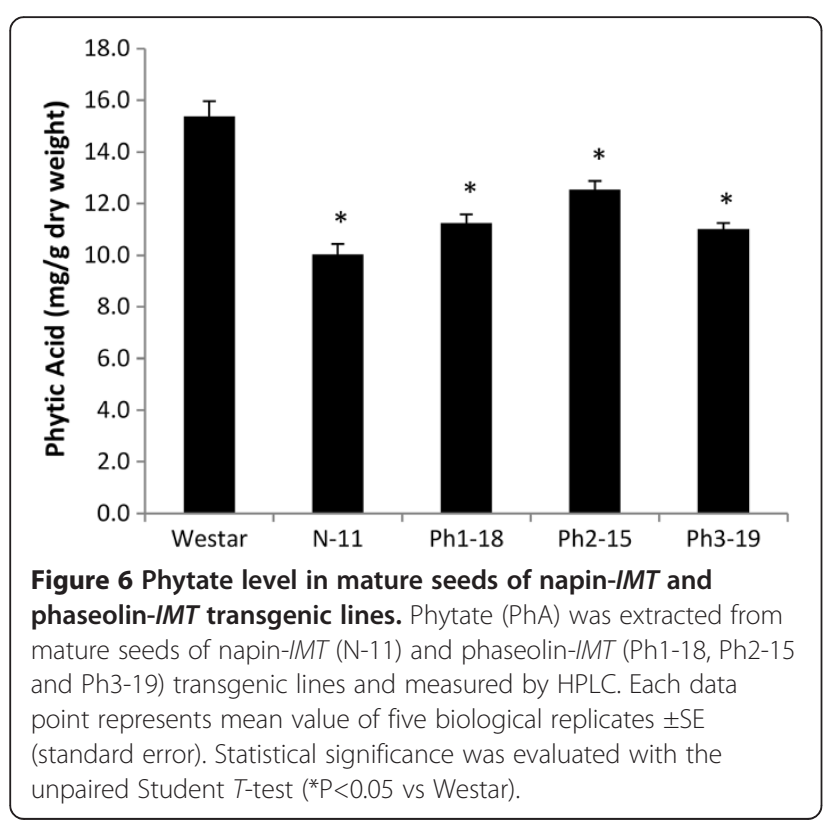

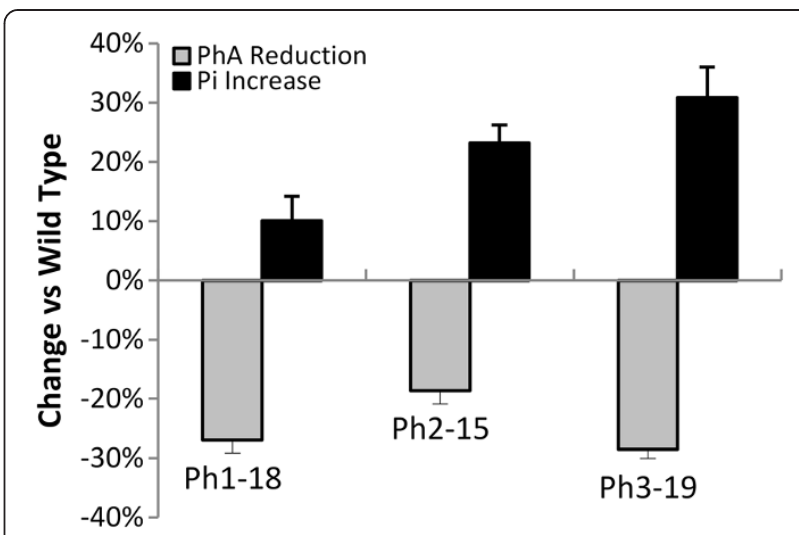

Figure 7 Changes in phytate and free phosphate in phaseolinIMT transgenic lines. Columns are expressed as percentage of reduction in phytate (PhA) (grey column) and increase in free phosphate $\left(\mathrm{P}_{\mathrm{i}}\right)$ (black column), \pm SE (standard error) in phaseolin-IMT transgenic lines, Ph1-18, Ph2-15 and Ph3-19.

competition for available Ins among three pathways (PhA, galactinol and ononitol production, Scheme 1). Such competition appears to have resulted in the reduction of galactinol biosynthesis with consequential downstream effects, which are reflected by increases in galactose and sucrose levels with a concomitant decrease in raffinose level. However, the decrease in raffinose level appears to be accompanied by a proportionate rise in stachyose (Figure 8).

\section{Discussion}

The relative incorporation of ${ }^{3} \mathrm{H}$-Ins in the different fractions of developing $B$. napus seeds, presented in Figure 1, depicts an image of the dynamics of Ins participation in phospholipid and PhA biosynthesis at early to mid stages of development. The data suggests the occurrence of horizontal interconversions between ${ }^{3} \mathrm{H}$-Ins and its polar and non-polar phosphorylated variants. Evidence of this is seen at 20 and 30 DAP where substantial shifts seem to occur across the hexane- and acid-soluble fractions.

The observed decrease in the relative contents of the acid-soluble fraction in the period 20-25 DAP reflects the utilization of myo-inositol in the biosynthesis of non-polar compounds (e.g. phospholipids). This is supported by the observation that a parallel increase in the relative amounts of label incorporation appeared in the hexane-soluble fraction during the same period. After 30 DAP, the relative rates of incorporation in the acidsoluble fraction increased again at the expense of the non-polar fraction, reflecting the rapid accumulation of PhA during this period (Figure 2). This is likely, since hydrolysis of Ins-containing phospholipids is known to lead to increased phytate accumulation in seeds of B. napus [11]. 


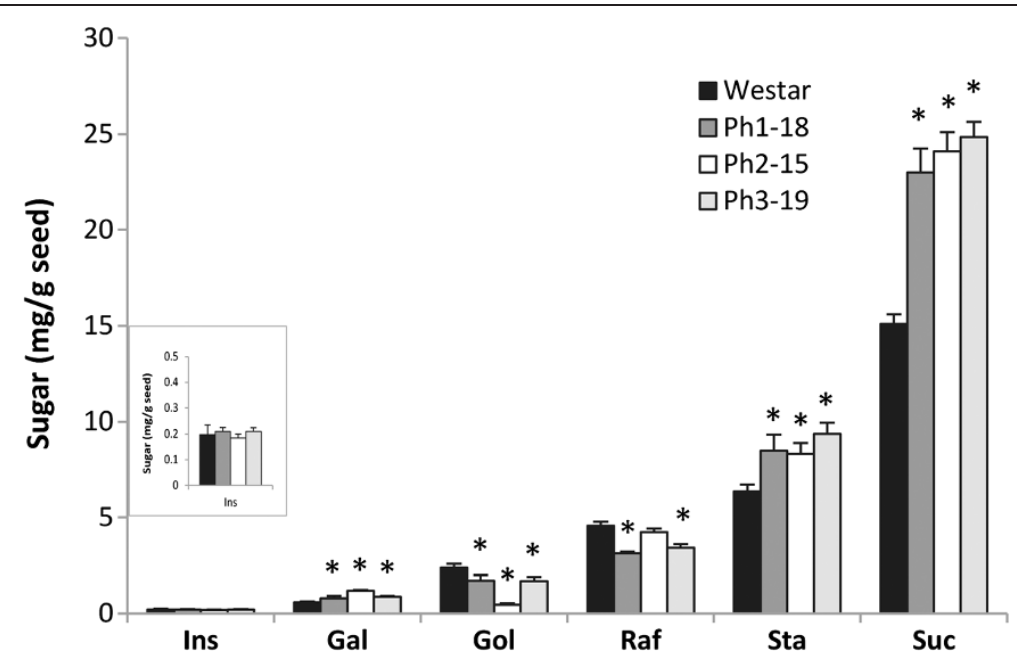

Figure 8 Sugar levels in mature seeds of phaseolin-IMT transgenic lines. Sugars were extracted from defatted mature seeds from wild-type Westar (black column) and phaseolin-IMT transgenic lines, Ph1-18 (dark grey), Ph2-15 (white) and Ph3-19 (light grey) and measured by HPLC. Each data point represents mean value of five biological replicates \pm SE (standard error). Statistical significance was evaluated with the unpaired Student T-test (*P<0.05 vs Westar). Ins, myo-inositol; Gal, galactose; Gol, Galactinol; Raf, Raffinose; Sta, Stachyose; Suc, Sucrose.

Typically, the newly forming seed coat is made up of four layers of cells, the outermost epidermal and palisade cell layers (which develop from the outer integument of the ovule) and parenchyma and endothelial cell layers (which are derived from the inner ovule integument) [27]. At 5 DAP the majority of seed inner space is filled with differentiating integument and a small amount of endosperm. That the majority of Ins at 15-20 DAP is localized in the seed coat of dissected seeds (Figure 3B) and that the bulk of the seed at 5-15 DAP is primarily made up of this tissue may be construed as evidence that the seed coat is the primary site of Ins biosynthesis in the B. napus seed. In support of this is the abundant supply of sucrose, which is transported through the phloem, apoplastic region and the seed coat during this early stage $[28,29]$ and which, through hydrolysis, acts as source of G-6-P, the precursor for Ins.

As the embryo expands through its initial globular, heart, torpedo and bent cotyledon stages, the endosperm proportionally shrinks (Figure 3C), and Ins becomes localized mainly in the embryo and seed coat (Figure 3B). That the seed coat is the tissue where Ins is most abundant at the early stages of seed development is of special importance since it plays a major role in the biosynthesis of mucilage, other seed coat polysaccharides and sugar acids through its participation in the oxidative pathway [30]. PhA synthesis started at very early stages (<10DAP) and reached its maximum levels at about 35 DAP (Figure 2). Distribution analysis of PhA accumulation between cotyledons and embryo axes revealed that from 20 to 75 DAP more than $80 \%$ of $\mathrm{PhA}$ is accumulated in cotyledons (80\% at 20-25 DAP, increasing up to $90 \%$ after 30 DAP) (data not shown). At 25-30 DAP, as the Brassica embryo expanded, higher accumulation of the non-polar derivatives (needed for membrane biogenesis) occurred (Figure 1). This period (25-30 DAP) appears to be marked by a slower decline in Ins levels, nearly reaching a plateau (Figure 3A), while, in parallel, PhA levels continued to rise at a relatively slower rate. After 25 DAP and through Ins appears to have reached a stable level in embryo, and more so in seed coat which could account for its apparent slower decline between 25 and 30 DAP (Figure 3B). At this stage the decline in Ins levels was accompanied by a concomitant rise in PhA levels (Figure 2) at the expense of the non-polar components (Figure 1) as the seed approached desiccation through the onset of RFO biosynthesis (30-35DAP) (unpublished results).

Since the initial phosphorylation steps of free Ins commence either with the reconstitution of L-Ins-1-P or through other positional phosphate esters, our strategy was to investigate the effect of competitive metabolic shunt of Ins on its phosphorylation and subsequent $\mathrm{PhA}$ and RFO accumulation in canola seeds. To accomplish this, we have explored the conversion of Ins into ononitol (1-D-4-O-methyl-myo-inositol), by methylation at the D-4 position, through the action of the IMT enzyme.

The observed variations in PhA reduction levels do not necessarily reflect different IMT expression levels since all selected lines displayed almost uniform levels of the IMT protein at the time of sampling as shown by Western blot analysis (Figure 4A and 4B). That the recorded ranges of $\mathrm{PhA}$ reduction appear to be similar with either promoter (Figure 6) suggests one of two possible scenarios: a) A certain threshold may exist at which a steady balance between supply and removal of Ins is reached, which confines $\mathrm{PhA}$ reduction levels within the 
observed limits; b) The temporal appearance of IMT activity under these promoters may not be in synchrony with the highest point of Ins accumulation, which is presumably reached in less than 10 DAP (Figure 3A), leading to the early onset of PhA synthesis. The latter scenario is likely, since the IMT protein was not detected until 25 DAP in lines Ph1-18 and Ph2-15 (Figure 4A) in contrast to PhA accumulation which is shown to be in progress at 15-20 DAP (Figure 2). A similar result was obtained when a MIPS antisense transcript was expressed in transgenic rice driven by the glutelin GluB-1 promoter [17].

The phaseolin promoter was chosen based on its reported early transcriptional activation in transgenic systems such as tobacco (15-16 DAP) [31,32]. The napin promoter which is native to Brassica was chosen with a view to comparing the effect of temporal expression differences of the two promoters on PhA accumulation. However, because IMT transcription from the phaseolin promoter did not commence as early as in the case of transgenic tobacco, it was not possible to assess such differences. The complex architecture of the phaseolin promoter has been shown to play a major role in spatial regulation of this promoter in transgenic systems [33]. Further, seed-specific transcriptional regulatory regions in the same promoter have been identified which affect its activation in a temporally dependent manner $[34,35]$. Therefore, it is possible that the phaseolin promoter, when expressed in different systems, could be affected by elements that may impose temporal expression variations. This is suggested by the fact that, in our hands, the transcriptional activation of phaseolin$I M T$ was triggered in canola at a later time point (20 DAP, Figure $4 \mathrm{~A}$ ) than in tobacco, indicating that this promoter could be influenced by developmentally regulated programs in a host-specific manner.

Attempts at enhancing the IMT translation efficiency through the modification of its translational context $[36,37]$ resulted in somewhat improved translation levels at 20 DAP under the phaseolin promoter when a dA nucleotide was positioned at the -3 position and a $\mathrm{dC}$ nucleotide at each of positions -2 and -1 of the initiation codon (transgenic line Ph3-19, Table 1 and Figure 4A). Translation levels at subsequent stages in the same line also appear to have been relatively enhanced. However, in spite of the protein level enrichment at 20 DAP in line Ph3-19, this did not substantially improve the overall $\mathrm{PhA}$ reduction, confirming the need for a more temporally and (or) spatially synchronized IMT expression and Ins production, as opposed to early enhancement of IMT translation. Nevertheless, while the apparent compartmentalization of Ins in the seed coat may shield it from IMT action in the embryo, seed-specific promoters are known to drive gene expression in the inner layer of seed coats and there is active transport of Ins from seed coat to embryo [38].

In mutant lines of other crops (e.g. maize [13,39] and soy bean [15]), the decrease in PhA phosphorous in mature seeds is generally accompanied by a parallel, albeit variable, increase in $P_{i}$ [15]. However, in some mutant lines (e.g. maize lpa2-1), the rise in $P_{i}$ can be accompanied by an accumulation of other Ins phosphates $\left(\operatorname{Ins}(1,2,4,5,6) \mathrm{P}_{5} ; \operatorname{Ins}(1,4,5,6) \mathrm{P}_{4} ;\right.$ and $\left.\operatorname{Ins}(1,2,6) \mathrm{P}_{3}\right)$ [39]. This makes the decrease in PhA futile in such mutants since these highly phosphorylated Ins species retain many of the adverse PhA properties. In the present study, although we observed a similar inverse relationship between PhA and $P_{i}$, there was no detectable accumulation of any of the partially phosphorylated Ins intermediates such as $\operatorname{InsP}_{3}, 4$ or 5. Furthermore, overexpression of IMT did not affect the seed viability or the germination efficiency of transgenic canola seeds. In addition, there were no measurable yield penalties (Table 2).

Absence of ononitol in wild-type HPLC chromatograms and failure of the wild-type protein extracts to produce ononitol, together with the Western analysis results suggest possible absence of native IMT-like activity in B. napus.

In mature transgenic seeds, the level of Ins was not changed significantly (Figure 8 ) but presumably during maturation was partitioned among PhA, galactinol and ononitol biosyntheses (Figure 5B). Accordingly, PhA formation was decreased in concert with ononitol accumulation. This pathway perturbation (Scheme 1) would have lowered the production of galactinol as well, and consequently, RFO accumulation [40] with the notable increase in sucrose levels (Figure 8). Curiously, the decrease in raffinose levels was accompanied by an almost proportionate rise in stachyose. Since sucrose levels remained high in transgenic seeds, the inverse modulation in the levels of raffinose and stachyose could indicate raffinose as a possible galactosyl donor for the subsequent chain elongation in a raffinose:raffinose galactosyltransfer manner, in which a molecule of raffinose would release its sucrose moiety after each galactosyl-residue transfer to another raffinose molecule

\section{Table 2 Seed yield of IMT transgenic lines}

\begin{tabular}{ll}
\hline Line & Seed yield (g/plant) \\
\hline Westar & $10.56 \pm 0.76$ \\
N-11 & $11.20 \pm 0.80$ \\
Ph1-18 & $10.11 \pm 0.62$ \\
Ph2-15 & $9.74 \pm 0.69$ \\
Ph3-19 & $9.76 \pm 0.69$ \\
\hline
\end{tabular}

Mature seeds of Westar, napin-IMT (N-11) and phaseolin-IMT (Ph1-18, Ph2-15 and Ph3-19) transgenic lines were harvested and weighed. Each data point represents mean value of five biological replicates $\pm S E$ (Standard error). 
(Figure 9B) [41,42]. This is conceivable in view of the limited availability of galactinol (Figure 8). Alternatively, since methylated derivatives of galactinol are known to take part in RFO synthesis in some plants [43], and although not hitherto proven in B. napus, we propose that ononitol, the methylated product of IMT, may be utilized to form the corresponding galactosylononitol (methylated galactinol), which could still participate, in part, in RFO-chain elongation to the higher oligomer, stachyose (Figure 9A). Since this does not account for the observed increase in sucrose levels, we postulate that both routes may be working concertedly. The differential extent of stachyose and sucrose accumulation (Figure 8) could be indicative of the different kinetics of the two routes (Figure 9A and 9B).

\section{Conclusions}

Early stages of Brassica seed development appear to be dominated by reciprocal interconversions of polar Ins phosphates and non-polar species. Although Ins is shown to be produced in significant amounts in the endosperm as well as seed coat during the early stages, no PhA accumulation occurs in those tissues. Instead, $\mathrm{PhA}$ accumulation appears to be mainly restricted to the embryo throughout seed development and maturation.

In addition to lowering PhA levels in the developing seeds, the competitive methylation of Ins resulted in

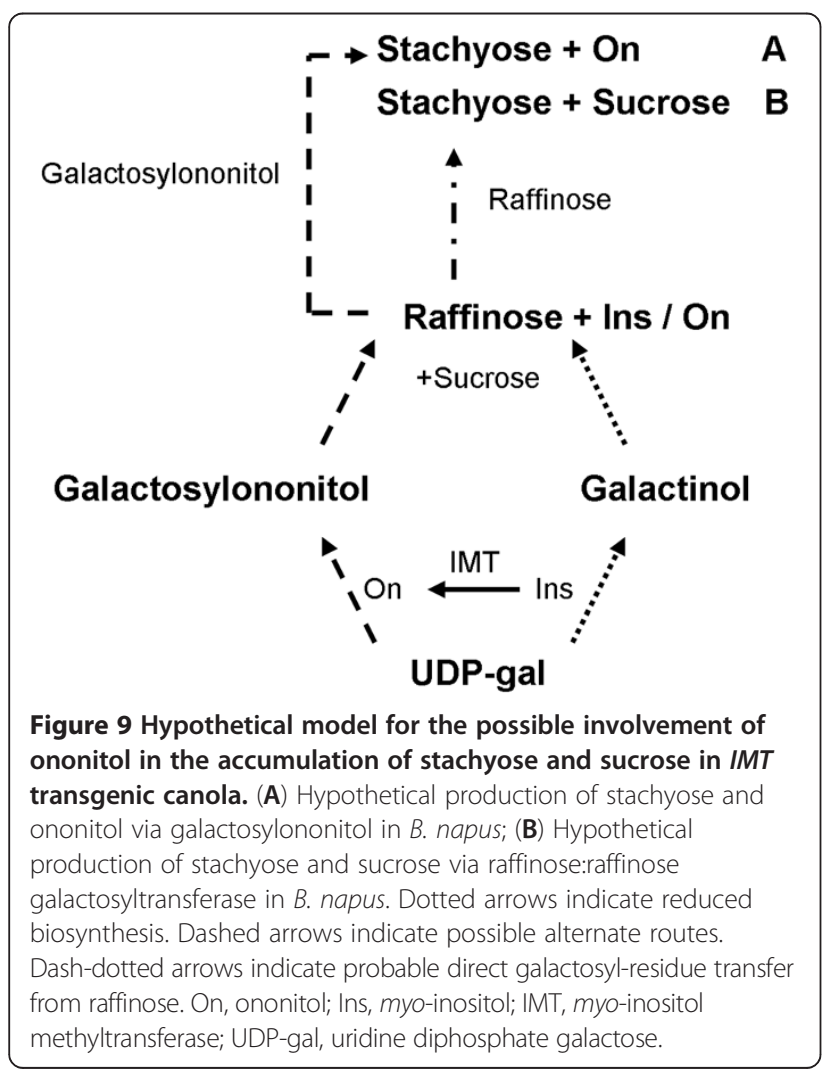

changes in the distribution and accumulation patterns of seed carbohydrates, leading to enhancement of the digestible and metabolizable energy profile of the meal as demonstrated by the higher content of the nutritionally useful sugar, sucrose. While the ratio of raffinose to stachyose was altered, the overall balance of RFO appears to be unaffected. No deleterious effects were encountered as a result of Ins methylation in developing Brassica seed.

It is evident from this study as well as previous ones that one of the answers to reducing phytate in crop seeds to a level which allows an appreciable beneficial effect on phosphorous and microelements bioavailability without adversely affecting yield and phenotype may be found in a model which combines the additive effects of more than one mechanism acting concertedly with separate independent contributions. Such an approach would allow selective inhibition of Ins phosphorylation at different steps for optimum phytate reduction. Nonetheless, the current strategy, if adopted, could potentially raise the canola market value as well as that of other crops.

\section{Methods}

Plant material and chemicals

Ice plant (Mesembryanthemum crystallinum) seeds were germinated, grown, salt treated and sampled as described previously $[20,44]$. Seeds of B. napus (Westar) plants, grown under growth chamber conditions (16 hour day at $20^{\circ} \mathrm{C} / 8$ hour night at $15^{\circ} \mathrm{C}$ photoperiod) were harvested at maturation. Seeds at different developing stages were also collected. Developing seeds were separated into seed coats and embryos on ice under a binocular dissecting microscope. Fresh seeds were cut half open with a scalpel. Endosperm of 15 and 20 DAP was collected by pipette. The seed coat and embryos were washed with ultra pure water three times. The excess water was absorbed with filter paper. The developing seeds and dissected tissue were frozen immediately in liquid $\mathrm{N}_{2}$. These were freeze dried for 24 hours and then extracted for PhA and Ins analysis.

Ononitol standards were purchased from GlycoSyn Technologies, Lower Hutt, New Zealand.

In Vivo labeling of developing seeds with ${ }^{3} \mathrm{H}$-myo-inositol Siliques at different developmental stages (15-40 DAP) were cut from plants and the cut end immediately put into $10 \mathrm{ml}$ sterile culture medium in $50 \mathrm{ml}$ tubes supplemented with $5 \mu \mathrm{Ci}{ }^{3} \mathrm{H}$-myo-inositol and incubated in a growth chamber for two days. Seeds were harvested and crushed in liquid nitrogen then extracted consecutively with hexanes (lipid fraction), $0.5 \mathrm{~N} \mathrm{HCl}$ (phytate-containing acidsoluble fraction) and trifluoroacetic acid (TFA-soluble cell debris fraction). The radioactivity in each fraction was assessed in a scintillation counter. 


\section{Phytic acid extraction}

Mature canola seeds $(350 \mathrm{mg}$ ) were homogenized in $4 \mathrm{ml}$ of ammonia in methanol $(10 \% \mathrm{w} / \mathrm{w})$ [45], then vortexed. After a 10-minute incubation, $3 \mathrm{ml}$ of hexanes was added and the samples were vortexed and centrifuged (RCF 2500). The liquid phases were discarded and the seed pellets re-extracted with $3 \mathrm{ml}$ of hexanes, centrifuged and the supernatants discarded. Pellets were washed three times with $5 \mathrm{ml}$ of absolute methanol, resuspended in $6 \mathrm{ml}$ of $0.5 \mathrm{~N} \mathrm{HCl}$ and kept at room temperature for 15 minutes. The slurry was centrifuged and the supernatant was filtered through a $0.45 \mu \mathrm{m}$ GHP Acrodisk filter (Gelman Science) prior to HPLC analysis. At this stage samples were stored at $-20^{\circ} \mathrm{C}$. PhA was extracted from $50-100 \mathrm{mg}$ of seeds at different developmental stages. For dissected material, seed coat $(20 \mathrm{mg})$, endosperm $(20 \mathrm{mg})$, embryo: 15 DAP (10 mg), 20 DAP (50 mg) and older (100 mg) was used. The extraction volume of $\mathrm{HCl}$ was adjusted proportionately.

\section{HPLC conditions for phytic acid analysis}

Phytic acid analysis was performed on a Waters 660E multi-solvent delivery system equipped with in-line degasser AF, 717plus Autosampler and a Sedex 55 (S.E. D.E.R.E.) evaporative light scattering detector at $50^{\circ} \mathrm{C}, 2$ bar, gain 7. The HPLC system was controlled and data processed by the Waters Millennium ${ }^{\mathrm{Tm}} 2010$ Chromatography Manager, version 2.15.01. Samples were chromatographed on an IC-Pak Anion HC, $150 \mathrm{~mm} \times$ $4.6 \mathrm{~mm}$ column (WAT026770, Waters), at $22^{\circ} \mathrm{C}$, with $100 \mathrm{mM}$ nitric acid at a flow rate of $1.0 \mathrm{ml} / \mathrm{min}$. A Waters IC-Pak Anion Guard-Pak (WAT010551) was used as the pre-column. A fritted filter guard (A-103X Rep Frit (BLK) $0.94 \times 0.25$ from Upchurch Scientific) was placed in front of the pre-column. Injections, typically $75 \mu \mathrm{l}$ of undiluted sample, were in duplicate with a separate result generated for each injection. A calibration curve was prepared for each run with the levels of standards at 25, 50, 75 and $100 \mu \mathrm{g}$. Standards were prepared with phytic acid dodecasodium salt $\mathrm{C}_{6} \mathrm{H}_{6} \mathrm{O}_{24} \mathrm{P}_{6} \mathrm{Na}_{12} \cdot 9 \mathrm{H}_{2} \mathrm{O}$ (Sigma), which was dissolved in the same concentration $\mathrm{HCl}$ as that used to extract the samples $(0.5 \mathrm{~N})$ to a concentration of $10 \mu \mathrm{g} / \mu \mathrm{l}$. The concentrated solution was diluted to $1.0 \mu \mathrm{g} / \mu \mathrm{l}$ in $0.5 \mathrm{~N} \mathrm{HCl}$. Volumes of 25, 50, 75 and $100 \mu \mathrm{l}$ of the diluted standard were injected in duplicate and phytic acid was detected at an average retention time of 3.9 minutes.

\section{HPLC conditions for carbohydrate analysis}

Sugars were extracted from seeds, and analyzed by HPLC as described [40]. Briefly, duplicates of defatted tissue of ten seeds, unless otherwise indicated, were extracted with $80: 20 \mathrm{v} / \mathrm{v}$ ethanol-water at $70^{\circ} \mathrm{C}$ for $30 \mathrm{~min}$, followed by centrifugation and evaporation of the supernatant to dryness. Samples were reconstituted in 18-M $\Omega$ water, and filtered through $0.45 \mu \mathrm{m}$ nylon filters prior to HPLC analysis. To accommodate analysis of early seed stages, extraction and reconstitution volumes were proportional to sample weight.

Galactinol, myo-inositol and D-ononitol were separated on a CarboPac ${ }^{\text {Tn }}$ MA1 column $(4 \mathrm{~mm} \times 250 \mathrm{~mm})$ preceded by a CarboPac ${ }^{\text {Tu }}$ MA1 guard column $(4 \mathrm{~mm} \times 50 \mathrm{~mm})$ with $500 \mathrm{mmol} \mathrm{L}^{-1}$ isocratic $\mathrm{NaOH}$ at $0.40 \mathrm{~mL} \mathrm{~min}^{-1}$ as eluent and detected by high performance anion exchangepulsed amperometric detection (HPAE-PAD) using a Dionex ICS-3000 system (Dionex Corp., Sunnyvale, Calif.). Standards were galactinol, myo-inositol (SigmaAldrich, St. Louis, MO) and D-ononitol (GlycoSyn Technologies, Lower Hutt, New Zealand).

Glucose, galactose, fructose, sucrose, stachyose and raffinose were separated on a CarboPac ${ }^{\text {Tm }}$ PA1 column $(2 \mathrm{~mm} \times 250 \mathrm{~mm})$ preceded by a CarboPac ${ }^{\text {ru }}$ PA1 guard column $(2 \mathrm{~mm} \times 50 \mathrm{~mm})$ and then an Amino-Trap ${ }^{\text {tox }}$ guard column $(2 \mathrm{~mm} \times 50 \mathrm{~mm})$ with $25 \mathrm{mmol} \mathrm{L}^{-1}$ isocratic $\mathrm{NaOH}$ at $0.25 \mathrm{~mL} \mathrm{~min}^{-1}$ as eluent and detected by HPAE-PAD. Standards were galactose, fructose, sucrose, raffinose, stachyose (Sigma-Aldrich, St. Louis, $\mathrm{MO}$ ), and glucose (Fisher Scientific, Hampton, NH).

\section{Inorganic phosphate analysis}

Inorganic phosphate $\left(\mathrm{P}_{\mathrm{i}}\right)$ in mature transgenic seeds was assayed using published protocols [46].

\section{Cloning of $I M T$ and plant transformation}

Fresh salt-stressed ice plant leaf tissue was frozen in liquid nitrogen and crushed to fine powder. Total RNA was extracted with TRIzol $^{\circ}$ Reagent (Invitrogen). Poly (A) ${ }^{+}$ RNA was isolated using published methods [47]. The first strand cDNA was generated using the Roche reverse transcription kit, and amplified with the sequence specific primers (forward, 5'-TTTTTGGATCCAAGAGAA AAA AAAATGACTACTTACACC-3' and reverse, 5'-TTTTTG CGGCCGCATAAAGGCAAATCATACACTG-3') by PCR based on the published sequence (Accession No. M87340). The reaction was initiated by heating at $94^{\circ} \mathrm{C}$ for $2 \mathrm{~min}$ followed by 35 cycles of heating at $94^{\circ} \mathrm{C}$ for $1 \mathrm{~min}$, annealing at $52^{\circ} \mathrm{C}$ for $1 \mathrm{~min}$ and extension for $3 \mathrm{~min}$ at $72^{\circ} \mathrm{C}$. The PCR product (1418bp) was purified using a PCR purification kit (Promega) followed by digestion with BamHI and NotI, whose sites were incorporated into the forward and reverse primers, respectively. The digested DNA fragment was subcloned into pSPORT1 (Invitrogen) and sequenced. The IMT gene was subcloned into pRD400 [48], which contains a napin promoter to produce plasmid pNIMT. Additional versions of the IMT gene, with modified translational context were produced 
[36,37] (Table 1). These as well as the parent-transgene were cloned into pRD 400 under the phaseolin promoter to generate pPhIMT1-3. All constructs were transferred into Agrobacterium tumefaciens strain GV3101 containing the helper plasmid pMP90 by triparental mating, followed by Agrobacterium-mediated transformation of Brassica napus (cv. Westar) [49].

\section{Over-expression of IMT in E coli and production of antibodies}

The IMT cDNA fragment was cloned into the bacterial expression plasmid, pPROEXHTb (Invitrogen). Protein expression was induced by adding IPTG to the culture medium to a final concentration of $1 \mathrm{mM}$. The $E$. coli culture was harvested after a 3 -hour incubation at $37^{\circ} \mathrm{C}$. The His-tagged protein was purified with Ni-NTA Agarose (Qiagen) under denaturing conditions. The purified protein $(43 \mathrm{Kda})$ was used to raise polyclonal antibodies against the IMT enzyme [50], which were subsequently used in Western analyses of transgenic lines.

\section{Southern and Northern blot analyses}

Genomic DNA was extracted from leaf tissue using Wizard ${ }^{\circ}$ Genomic DNA Purification Kit (Promega). RNA extraction from developing seeds of both transgenic and wild-type plants was conducted by using RNeasy plant total RNA kit (Qiagen). Southern and Northern analyses using Hybond- $\mathrm{N}^{+}$membrane (Amersham) were performed essentially as described [51].

\section{IMT Enzyme assay and Western analysis in transgenic plants}

Total protein extracts from developing seeds (approximately $40 \mathrm{DAP}$ ) as well as leaves of transgenic lines, and wild-type plants were assayed for IMT-enzyme activity exactly as described previously [20].

For Western analysis, total soluble proteins were extracted from developing seeds as described [44]. TND buffer, $90 \mathrm{mM}$ Tris- $\mathrm{HCl}\left(\mathrm{pH} 8.3\right.$ at $\left.4^{\circ} \mathrm{C}\right), 9 \mathrm{mM}$ DTT and $2 \mathrm{mM}$ Leupeptin $(100 \mu \mathrm{l})$ was added to $50 \mathrm{mg}$ of frozen seeds crushed in liquid nitrogen. Soluble protein samples were prepared by collecting the supernatants. Protein concentration in each sample was determined using the Bradford assay with BSA as the standard. Protein samples $(15 \mu \mathrm{g}$ each) were used in Western blot analysis. The samples were separated by PAGE and blotted on a Nitrocellulose membrane (Bio-Rad) [51]. The immunereactions were conducted using Bio-Rad Immun-Blot ${ }^{\circledR}$ Assay Kit.

\section{Abbreviations}

DAP: Days after pollination; Ptd-CMP: Phosphatidylcytosine monophosphate; Ptdlns: Phosphatidylinositol; PI-K: Phosphatidylinositol kinase; PtdlnsP: Phosphatidylinositol monophosphate; GolS: Galactinol synthase; RFO: Raffinose oligosaccharides.

\section{Competing interests}

The authors declare that they have no competing interests.

\section{Authors' contribution}

FG, project PI, designed the concept, supervised all the experiments, contributed to and edited the manuscript. JD, WY, CB, KN performed the experiments and participated in the interpretation of results. WK supervised Brassica transformation experiments. All authors read and approved the final manuscript.

\section{Acknowledgements}

This study was supported through an NRCC-DASCI Strategic Alliance Agreement. Ice plant seeds were a gift from Professor Hans Bohnert. The phaseolin promoter was provided by Dow AgroSciences as pAGM219 plasmid. The contributions of Atta Hussain, supported by grants from the Canola Council of Canada and Dow AgroSciences, are hereby acknowledged. We also thank Sandra Polvi for technical support. This is NRCC publication number 54672 .

\section{Author details}

${ }^{1}$ Wilmar International, 56 Neil Rd, Singapore 088830, Singapore. ${ }^{2}$ National Research Council Canada, Plant Biotechnology Institute, 110 Gymnasium Place, Saskatoon, SK S7N 0W9, Canada. ${ }^{3}$ Ag-West Bio Inc, 101 - 111 Research Drive, Saskatoon, SK S7N 3R2, Canada.

Received: 14 December 2012 Accepted: 9 May 2013

Published: 21 May 2013

\section{References}

1. Luley-Goedl C, Nidetzky B: Glycosides as compatible solutes: biosynthesis and applications. Nat Prod Rep 2011, 28:875-896.

2. Vucenik I, Shamsuddin AM: Protection against cancer by dietary IP6 and inositol. Nutr Canc 2006, 55:109-125.

3. Shamsuddin AM, Vucenik I, Cole KE: IP6: a novel anti-cancer agent. Life Sci 1997, 61:343-354.

4. Murphy AM, Brearley OB CA, Carr JP, Hanke DE: A role for inositol hexakisphosphate in the maintenance of basal resistance to plant pathogens. Plant J 2008, 56:638-652.

5. Schröterová L, Hasková P, Rudolf E, Cervinka M: Effect of phytic acid and inositol on the proliferation and apoptosis of cells derived from colorectal carcinoma. Oncol Rep 2010, 23:787-793.

6. Verghese M, Rao DR, Chawan CB, Walker LT, Shackelford L: Anticarcinogenic effect of phytic acid (IP6): Apoptosis as a possible mechanism of action. LWT 2006, 39:1093-1098.

7. Xu Q, Kanthasamy AG, Reddy MB: Neuroprotective effect of the natural iron chelator, phytic acid in a cell culture model of Parkinson's disease. Toxicology 2008, 245:101-108.

8. Macbeth MR, Schubert HL, VanDemark AP, Lingam AT, Hill CP, Bass BL: Inositol hexakisphosphate is bound in the ADAR2 core and required for RNA editing. Science 2005, 309:1534-1539.

9. Graf E, Eaton JW: Antioxidant functions of phytic acid. Free Radic Biol Med 1990, 8:61-69.

10. Doria E, Galleschi L, Galucci L, Pinzino C, Pilu R, Cassani E, Nielsen E: Phytic acid prevents oxidative stress in seeds: evidence from a maize (Zea mays L.) low phytic acid mutant. J Exp Bot 2009, 60:967-978.

11. Georges F, Das S, Ray H, Bock C, Nokhrina K, Kolla VA, Keller K: OverExpression of Brassica napus Phosphatidylinositol-Phospholipase C2 (BnPtdlns-PLC2) in Canola Induces Significant Changes in Gene Expression and Phytohormone Distribution Patterns, Enhances Drought Tolerance and Promotes Early Flowering and maturation. Plant Cell Environ 2009, 32:1664-1681.

12. Suzuki M, Tanaka K, Kuwano M, Yoshida KT: Expression pattern of inositol phosphate-related enzymes in rice (Oryza sativa L.): implications for the phytic acid biosynthetic pathway. Gene 2007, 405:55-64.

13. Pilu R, Panzeri D, Gavazzi G, Rasmussen SK, Consonni G, Nielsen E: Phenotypic, genetic and molecular characterization of a maize low phytic acid mutant (Ipa241). Theor App/ Genet 2003, 107:980-987.

14. Panzeri D, Cassani E, Doria E, Tagliabue G, Forti L, Campion B, Bollini R, Brearley CA, Pilu R, Nielsen E, Sparvoli F: A defective ABC transporter of the MRP family, responsible for the bean Ipa1 mutation, affects the 
regulation of the phytic acid pathway, reduces seed myo-inositol and alters ABA sensitivity. New Phytol 2011, 191:70-83.

15. Wilcox J, Premachandra G, Yound K, Raboy V: Isolation of high seed inorganic P, low-phytate soybean mutants. Crop Sci 2000, 40:1601-1605.

16. Kuwano M, Ohyama A, Tanaka Y, Mimura T, Takaiwa F, Yoshida KT: Molecular breeding for transgenic rice with low-phytic-acid phenotype through manipulating myo-inositol 3-phosphate synthase gene. $\mathrm{Mol}$ Breed 2006, 18:263-272.

17. Kuwano M, Takaiwa F, Yoshida KT: Differential effects of a transgene to confer low phytic acid in caryopses located at different positions in rice panicles. Plant Cell Physiol 2009, 50:1387-1392.

18. Nunes ACS, Vianna GR, Cuneo F, Guy de Capdeville JA-F, Rech EL, Aragão FJL: RNAi-mediated silencing of the L-myo-inositol-1-phosphate synthase gene (GmMIPS1) in transgenic soybean inhibited seed development and reduced phytate content. Planta 2006, 224:125-132.

19. Georges F, Hussain AAK, Keller WA: Method for reducing phytate in canola meal using genetic manipulation involving myo-inositol 1-phosphate synthase gene. Patent 2006. WO 00/73473 A1

20. Vernon DM, Bohnert HJ: A novel methyl transferase induced by osmotic stress in the facultative halophyte Mesembryanthemum crystallinum. EMBO J 1992, 11:2077-2085.

21. Keller WA, Datla R, Dong J-Z, Georges F, Hussain AAK, Selvaraj G: Methods and compositions for modifying levels of secondary metabolic compounds in plants. Patent 2007. US7279619.

22. Vernon DM, Tarczynski MC, Jensen RG, Bohnert HJ: Cyclitol production in transgenic tobacco. Plant J 1993, 4:199-205.

23. Sheveleva E, Chmara W, Bohnert HJ, Jensen RC: Increased Salt and Drought Tolerance by D-Ononitol Production in Transgenic Nicotiana tabacum L. Plant Physiol 1997, 115:1211-1219.

24. Chiera JM, Streeter JG, Finer JJ: Ononitol and pinitol production in transgenic soybean containing the inositol methyl transferase gene from Mesembryanthemum crystallinum. Plant Sci 2006, 171:647-654.

25. Patra B, Ray S, Richter A, Majumder AL: Enhanced salt tolerance of transgenic tobacco plants by co-expression of PcINO1 and McIMT1 is accompanied by increased level of myo-inositol and methylated inositol. Protoplasma 2010, 245:143-152.

26. Paulick MG, Bertozzi CR: The glycosylphosphatidylinositol anchor: $A$ complex membrane-anchoring structure for proteins. Biochemistry 2008, 47:6991-7000

27. Moise JA, Han S, Gudynait-Savitch L, Johnson DA, Miki BLA: Seed coats: Structure, development, composition and biotechnology. In Vitro Cell Dev Biol Plant 2005, 41:620-644.

28. Coelho CMM, Benedito VA: Seed development and reserve compound accumulation in common bean (Phaseolus vulgaris L.). Seed Sci Biotechnol 2008, 2:42-52.

29. Morley-Smith ER, Pike MJ, Findlay K, Köckenberger W, Hill LM, Smith AM, Rawsthorne S: The transport of sugars to developing embryos is not via the bulk endosperm in oilseed rape seeds. Plant Physiol 2008, 147:2121-2130

30. Loewus FA, Kelly S, Neufeld EF: Metabolism of myo-inositol in plants: Conversion to pectin, hemicellulose, D-xylose, and sugar acids. Biochemistry 1962, 48:421-425.

31. Altenbach SB, Pearson KW, Meeker G, Staraci LC, Sun SM: Enhancement of the methionine content of seed proteins by the expression of a chimeric gene encoding a methionine-rich protein in transgenic plants. Plant Mol Biol 1989, 13:513-522.

32. Sengupta-Gopalan C, Reichert NA, Barker RF, Hall TC, Kemp JD: Developmentally regulated expression of the bean $\beta$-phaseolin gene in tobacco seed. Proc Natl Acad Sci USA 1985, 82:3320-3324.

33. Chandrasekharan MB, Bishop KJ, Hall TC: Module-specific regulation of the b-phaseolin promoter during embryogenesis. Plant J 2003, 33:853-866

34. Burow MD, Sen P, Chlant CA, Murai N: Developmental control of the $\beta$-phaseolin gene requires positive, negative, and temporal seed-specific transcriptional regulatory elements and a negative element for stem and root expression. Plant J 1992, 2:537-548.

35. Kawagoe $Y$, Murai $N$ : Four distinct nuclear proteins recognize in vitro the proximal promoter of the bean seed storage protein beta-phaseolin gene conferring spatial and temporal control. Plant J 1992, 2:927-936.

36. Kozak M: At least six nucleotides preceding the AUG initiator codon enhance translation in mammalian cells. Mol Biol 1987, 196:947-950.
37. Rangan L, Vogel C, Srivastava A: Analysis of Context Sequence Surrounding Translation Initiation Site from Complete Genome of Model Plants. Mol Biotechnol 2008, 39:207-213.

38. Stalberg $K$, Ellerstrom M, Josefsson LG, Rask L: Deletion analysis of a $2 S$ seed storage protein promoter of Brassica napus in transgenic tobacco. Plant Mol Biol 1993, 23:671-683.

39. Raboy V, Gerbasi PF, Young KA, Stoneberg SD, Pickett SG, Bauman AT, Murthy PP, Sheridan WF, Ertl DS: Origin and seed phenotype of maize low phytic acid 1-1 and low phytic acid 2-1. Plant Physiol 2000, 124:355-368.

40. Bock C, Ray H, Georges F: Down-regulation of galactinol synthesis in oilseed Brassica napus leads to significant reduction of antinutritional oligosaccharides. Botany 2009, 87:597-603.

41. Hopf H, Gruber G, Zinn A, Kandler O: Physiology and biosynthesis of lychnose in Cerastium arvense. Planta 1984, 162:283-288.

42. Vanhaecke M, Dyubankova N, Lescrinier E, Van den Ende W: Metabolism of galactosyl-oligosaccharides in Stellaria media - Discovery of stellariose synthase, a novel type of galactosyltransferase. Phytochemistry 2010, 71:1095-1103

43. Peterbauer T, Richter A: Galactosylononitol and Stachyose Synthesis in Seeds of Adzuki Bean. Plant Physiol 1998, 117:165-172.

44. Ostrem JA, Olsen SW, Scmitt JM, Bohnert HJ: Salt stress increases the level of translatable mRNA for phosphoenolpyruvate carboxylase in Mesembryanthemum crystallinum. Plant Physio/ 1987, 84:1270-1275.

45. Naczk M, Wanasundara PKJP, Shahidi F: Facile spectrotophotometric quantification method of sinapic acid in hexane-extracted and methanolammonia-water treated mustard and rapeseed meals. J Agric Food Chem 1992, 40:444-448.

46. Shi J, Wang H, Wu Y, Hazebroek J, Meeley RB, Ertl DS: The maize low-phytic acid mutant lpa2 is caused by mutation in an inositol phosphate kinase gene. Plant Physiol 2003, 131(2):507-515. Feb.

47. Aviv $H$, Leder P: Purification of biologically active globin messenger RNA by chromatography on oligothymidylic acid-cellulose. Proc Natl Acad SCi USA 1972, 69:1408-1412.

48. Datla RSS, Hammerlindl JK, Panchuk B, Pelcher LE, Keller W: Modified binary plant transformation vectors with the wild-type gene encoding NPTII Gene. Gene 1992, 211:383-384.

49. Moloney MM, Walker JM, Sharma KK: High efficiency transformation of Brassica napus using Agrobacterium vectors. Plant Cell Rep 1989, 8:238-242.

50. Nelson DE, Rammesmayer G, Bohnert HJ: Regulation of Cell-Specific Inositol Metabolism and Transport in Plant Salinity Tolerance. Plant Cell 1998, 10:753-764.

51. Sambrook J, Fritsch EF, Maniatis T: Molecular cloning, a laboratory manual, 3rd ED. Cold Spring Harbor Laboratory Press 1989, 1:6.39-7.50.

\section{doi:10.1186/1471-2229-13-84}

Cite this article as: Dong et al:: Perturbing the metabolic dynamics of myo-inositol in developing Brassica napus seeds through in vivo methylation impacts its utilization as phytate precursor and affects downstream metabolic pathways. BMC Plant Biology 2013 13:84.

\section{Submit your next manuscript to BioMed Central and take full advantage of:}

- Convenient online submission

- Thorough peer review

- No space constraints or color figure charges

- Immediate publication on acceptance

- Inclusion in PubMed, CAS, Scopus and Google Scholar

- Research which is freely available for redistribution 